\title{
Valores da pressão arterial em cães pelos métodos oscilométrico e Doppler vascular
}

[Arterial blood pressure in dogs by the oscilometric method and the Doppler ultrasonic]

\author{
R.R. Cabral ${ }^{1}$, B.D. Ciasca ${ }^{1}$, V.M.C. Oliveira ${ }^{2}$, A.P. Vaz-Curado ${ }^{2}$, M.H.M.A. Larsson $^{1 *}$ \\ ${ }^{1}$ Faculdade de Medicina Veterinária e Zootecnia - USP \\ Av. Professor Orlando Marques de Paiva, 87 \\ 05508-270 - São Paulo, SP \\ ${ }^{2}$ Aluno de pós-graduação - FMVZ-USP - São Paulo, SP
}

\begin{abstract}
RESUMO
Foram comparados dois métodos não-invasivos de medida da pressão arterial, o Doppler vascular e o oscilométrico, com o objetivo de estabelecer parâmetros que possam auxiliar no diagnóstico seguro da hipertensão arterial. Para tal, foram utilizados 45 cães, machos e fêmeas, distribuídos em três grupos de acordo com o peso, pequeno, médio e grande porte. Em cada animal, procedeu-se a mensuração por meio do Doppler vascular e, em seguida, do oscilométrico. Na obtenção da pressão arterial sistólica, não houve diferença entre os métodos nos três grupos de animais, porém, na obtenção da pressão arterial diastólica, houve diferença estatística entre o Doppler vascular e o oscilométrico nos grupos de animais de pequeno e médio porte. Pôde-se concluir que valores confiáveis de pressão sistólica podem ser obtidos tanto por meio do Doppler vascular quanto do oscilométrico. Os valores da pressão arterial diastólica obtidos pelos dois métodos não se correlacionam, principalmente nos animais de pequeno e médio porte.
\end{abstract}

Palavras-chave: cão, pressão arterial, oscilométrico, Doppler

\begin{abstract}
Two noninvasive methods for blood pressure measurement, the oscilometric and the Doppler ultrasonic, were compaired aiming the establishment of parameters that can be helpful for the hypertension diagnosis. Fortyfive dogs, males and females, were distributed in three groups, according to body weight: small, medium, and large sizes. In each animal, measurement of the blood pressure was performed, first by the Doppler ultrasonic, followed by the oscilometric method. No differences were observed in sistolic arterial pressure values obtained by both methods in none of the three animal groups. However, there was an important difference in diastolic arterial pressure values obtained by these methods, especially in small and medium size dogs. It was concluded that reliable results of sistolic blood pressure can be obtained both by the Doppler ultrasonic and the oscilometric method. Diastolic blood pressure results, however, showed no correlation between these two methods, mainly in small and medium size dogs.
\end{abstract}

Keywords: dog, pressure arterial, oscilometric, Doppler

\section{INTRODUÇÃO}

A hipertensão arterial sistêmica é uma enfermidade que afeta cães e gatos e vem, reconhecidamente, ganhando importância na prática da clínica veterinária (Jepson et al., 2005). Diferentes tecidos podem ser danificados pela hipertensão sistêmica. Há, por exemplo, uma forte relação entre injúria ocular e

Recebido em 28 de maio de 2008

Aceito em 28 de dezembro de 2009

*Autor para correspondência (corresponding author)

E-mail: akaolar@usp.br hipertensão em cães e gatos (Brown e Henik, 1998). O rim é outro órgão muito suscetível a danos causados pela hipertensão sistêmica. Quando a pressão elevada atinge diretamente os capilares glomerulares, ela causa hipertensão glomerular e consequente dano aos glomérulos, com redução progressiva da função renal (Brown et al., 1995). Outras manifestações secundárias à hipertensão arterial são hipertrofia concêntrica do ventrículo esquerdo, disfunção diastólica e 
insuficiência valvar secundária, devido ao aumento da resistência vascular periférica (Douglas e Tallant, 1991) e hemorragia cerebral (Brown e Henik, 1998).

$\mathrm{Na}$ clínica, o diagnóstico de hipertensão sistêmica é baseado na determinação da pressão sanguínea arterial. Tilley e Goodwin (2002) classificaram as pressões sanguíneas em quatro grupos diferentes, estabelecendo, dessa forma, parâmetros para sua avaliação clínica: normal pressão arterial sistólica (PAS) entre 110 a $120 \mathrm{mmHg}$ e pressão arterial diástólica (PAD) entre 70 a $80 \mathrm{mmHg}$; discretamente elevada PAS entre 120 a $170 \mathrm{mmHg}$ e PAD entre 80 a $100 \mathrm{mmHg}$; moderadamente elevada - PAS entre 170 a $200 \mathrm{mmHg}$ e PAD entre 100 a $120 \mathrm{mmHg}$; e acentuadamente elevada - PAS acima de $200 \mathrm{mmHg}$ e PAD acima de $120 \mathrm{mmHg}$.

A pressão sanguínea arterial pode ser avaliada tanto por métodos invasivos (diretos), quanto por métodos não-invasivos (indiretos). Muito embora os métodos invasivos ofereçam vantagens como a representação consistente e precisa da pressão sistêmica, sendo o padrão para a comparação de métodos indiretos (Podell, 1992), eles são, no entanto, raramente utilizados na prática, devido às dificuldades da técnica (Porciello et al., 2004), que incluem sedação ou anestesia do paciente, fatores que, além de pouco práticos, podem reduzir artificialmente a pressão arterial (Kittleson e Oliver, 1983). Por esses motivos, os métodos invasivos de medida da pressão arterial são mais usados em pesquisas (Chalifoux et al., 1985).

Os métodos não-invasivos, contudo, são preferivelmente utilizados em situações clínicas devido à maior praticidade de uso e à possibilidade de ser repetido entre pequenos intervalos de tempo. Entretanto, as técnicas indiretas são menos precisas quando a pressão sanguínea é baixa, quando há vasoconstrição ou quando ocorre movimentação excessiva do animal (Podell, 1992). Algumas classes de dispositivos não-invasivos para medir pressão sanguínea disponíveis atualmente são o Doppler vascular, esfigmomanômetro oscilométrico e aparelhos de fotopletismografia (Jepson et al., 2005).

Estudos já foram realizados para a avaliação de técnicas de medida da pressão arterial em cães, comparando diferentes métodos, com animais em diversos estados de consciência e em diferentes posições (Stepien e Rapoport, 1999). Bodey et al. (1996) compararam métodos diretos e indiretos (método oscilométrico) para medir a pressão arterial em cães conscientes e estabelecer uma faixa de normalidade. Um estudo posterior mostrou que a faixa de normalidade pode variar fisiologicamente nos cães, principalmente de acordo com a raça e a idade (Bodey e Michell, 1997). Trabalhos mostram que a pressão sanguínea varia em algumas doenças específicas, sendo normalmente mais elevada em cães com Diabetes mellitus (Bodey e Michell, 1997), hiperadrenocorticismo (Marco et al., 2000), cardiopatias e nefropatias (Larsson e D'Urso, 1998).

De acordo com Jepson et al. (2005), a pressão arterial sistólica pode ser aferida em $100 \%$ das tentativas de uso do Doppler em gatos conscientes. Em contrapartida, a pressão arterial diastólica pôde ser aferida em apenas $51,4 \%$ das tentativas de uso do Doppler. As médias de pressão arterial diastólica obtidas por dois examinadores, nesse caso, foram estatisticamente diferentes entre si, o que implica que a pressão arterial diastólica, obtida por meio do Doppler, pode não ser confiável. Já com relação à aferição desses parâmetros por meio do método oscilométrico, esses mesmos autores relatam maior dificuldade, uma vez que o animal teria de permanecer sem se mover durante todo $\mathrm{o}$ procedimento, o que nem sempre foi possível. Ao comparar os resultados obtidos entre o Doppler e o método oscilométrico, os autores não encontraram diferença significativa entre as médias de leitura da pressão sanguínea sistólica, porém, muito embora as médias de pressão arterial obtidas fossem comparáveis entre ambos os aparelhos, houve maior variação nos dados pontuais fornecidos pelo aparelho oscilométrico. Já com relação à pressão arterial diastólica, o método oscilométrico mostrou-se estatisticamente superior.

Frente à importância que a hipertensão arterial vem assumindo no cotidiano do médico veterinário, faz-se necessário conhecer aparelhos confiáveis que auxiliem no rápido diagnóstico dessa doença. Os métodos não-invasivos de avaliação da pressão arterial disponíveis atualmente, embora tenham seu uso já 
consagrado, ainda deixam muitas dúvidas quanto à sua acurácia e praticidade na rotina clínica. $\mathrm{O}$ objetivo deste trabalho foi comparar dois métodos não-invasivos, o oscilométrico e o Doppler vascular, para, dessa forma, estabelecer parâmetros que possam auxiliar no diagnóstico seguro da hipertensão arterial em cães.

\section{MATERIAL E MÉTODOS}

Foram utilizados 45 cães, machos e fêmeas, de dois a oito anos de idade, sendo 15 de pequeno porte, 15 de médio porte e 15 de grande porte. Foram considerados como animais de pequeno porte aqueles com peso de $0 \vdash 15 \mathrm{~kg}$ (grupo I); de médio porte, de $15-30 \mathrm{~kg}$ (grupo II); e de grande porte, peso igual ou acima de $30 \mathrm{~kg}$ (grupo III).

Realizou-se exame físico, que consistiu na determinação dos parâmetros vitais dos animais, por meio da determinação das frequências cardíaca e respiratória, frequência de pulso, temperatura corpórea e do tempo de preenchimento capilar, bem como avaliação de coloração de mucosas aparentes, grau de hidratação e de linfonodos palpáveis. O exame físico incluiu também exame torácico, por meio de inspeção, palpação, percussão dígito-digital e auscultação indireta; e exame do abdômen por palpação e percussão.

A determinação da pressão arterial foi realizada por meio de dois métodos não-invasivos: Doppler vascular e oscilométrico. Para o Doppler (Medmega, modelo DV610, Ind. Equip. Médicos Ltda - Franca, SP), os animais foram posicionados em decúbito lateral direito. Primeiramente, foi realizada tricotomia da região palmar metacarpal próxima ao coxim, onde o pulso era palpável. Aplicou-se gel sobre a região tricotomizada e sobre a superfície do transdutor do aparelho, que foi ali posicionado. Colocou-se um manguito (de largura correspondente a $40 \%$ da circunferência do membro) sobre o terço proximal da região radioulnar do membro torácico do cão. Após a obtenção dos sinais de pulso adequados, o manguito foi inflado até aproximadamente $30 \mathrm{mmHg}$ superior à pressão necessária para se obliterar o sinal de pulso audível e, em seguida, lentamente desinflado. A pressão sistólica foi determinada no momento em que o sinal de pulso se tornou audível novamente, e a pressão diastólica quando os sinais audíveis, abruptamente, diminuíram ou alteraram o timbre, tornando-se abafados. Já para o método oscilométrio (Dixtal, modelo DX2710, Biomédica Ind. Com. Ltda. - Manaus, AM), com os animais posicionados em decúbito lateral direito, o manguito (de largura correspondente a $40 \%$ da circunferência do membro) foi colocado sobre o terço proximal da região radioulnar do membro torácico do cão, e o membro do animal foi posicionado no nível do coração direito. O manguito foi, então, automaticamente inflado a uma pressão suprasistólica e, em seguida, desinflado de modo progressivo. Mensurações automáticas das pressões sistólica, diastólica e média foram obtidas em cinco ciclos, com intervalo de um minuto.

A avaliação e a comparação dos resultados foram realizadas por meio de análises estatísticas, com a utilização do programa "MINITAB 14". Utilizou-se o teste de normalidade de AndersonDarling, para saber se as amostras obedeciam ou não a uma distribuição normal. Os resultados foram expressos sob a forma de tabelas e figuras.

\section{RESULTADOS}

No grupo I, foram avaliados quatro $(26,7 \%)$ machos e 11 (73,3\%) fêmeas; no grupo II, cinco $(33,3 \%)$ machos e $10(66,7 \%)$ fêmeas; e no grupo III, seis $(40,0 \%)$ machos e nove $(60,0 \%)$ fêmeas. As médias de peso e de idade, na mesma ordem de citação dos grupos, foram, respectivamente: $6,67 \pm 2,64 \mathrm{~kg}$ e $5,73 \pm 2,37$ anos, $23,09 \pm 2,99 \mathrm{~kg}$ e $4,33 \pm 1,23$ anos e $42,57 \pm 16,13 \mathrm{~kg}$ e 4,66 $\pm 1,98$ anos.

Nas Tab. 1, 2 e 3, apresentam-se os valores individuais e as médias das pressões arterial sistólica e arterial diastólica, obtidos pelos métodos Doppler vascular e oscilométrico. A pressão arterial média foi de $90,52 \pm 17,73 \mathrm{mmHg}$ para o grupo I, $105,65 \pm 16,01 \mathrm{mmHg}$ para o grupo II e $167,82 \pm 19,45 \mathrm{mmHg}$ para o grupo III. 
Valores da pressão arterial em cães...

Tabela 1. Médias da pressão arterial sistólica e diastólica de cães de pequeno porte, aferidas pelos métodos Doppler vascular e oscilométrico

\begin{tabular}{ccccccc}
\hline & Pressão sistólica & & & \multicolumn{3}{c}{ Pressão diastólica } \\
\cline { 1 - 2 } \cline { 5 - 6 } animal & PASd & PASo & & animal & PADd & PADo \\
\hline 1 & 122 & 137,6 & 1 & 118 & 94,6 \\
2 & 102 & 103 & 2 & 112 & 95,2 \\
3 & 126 & 135,6 & 3 & 98 & 64,4 \\
4 & 102 & 123,2 & & 4 & 68 & 110,6 \\
5 & 141 & 129,4 & & 5 & 92 & 122,4 \\
6 & 128 & 121,8 & & 6 & 81 & 94,4 \\
7 & 152 & 123,6 & & 7 & 118 & 70 \\
8 & 156 & 137 & 8 & 59 & 66,6 \\
9 & 133 & 141 & 9 & 96 & 66 \\
10 & 144 & 135,2 & 10 & 114 & 88,6 \\
11 & 118 & 131,8 & 11 & 112 & 95,8 \\
12 & 99 & 105,6 & 12 & 106 & 70,6 \\
13 & 132 & 119,2 & 13 & 90 & 84,4 \\
14 & 126 & 139 & 14 & 108 & 109,6 \\
15 & 142 & 102,2 & 15 & 102 & 93,4 \\
MD & 128,2 & 125,68 & MD & 98,26 & 88,44 \\
DP & 19,04 & 18,50 & DP & 18,53 & 19,63 \\
\hline
\end{tabular}

PASd: médias de pressão arterial sistólica aferidas pelo Doppler vascular; PASo: médias de pressão arterial sistólica aferidas pelo método oscilométrico; PADd: médias de pressão arterial diastólica aferidas pelo Doppler vascular; PADo: médias de pressão arterial diastólica aferidas pelo método oscilométrico; MD: média; DP: desvio-padrão.

Tabela 2. Médias de pressão arterial sistólica e diastólica de cães de médio porte, aferidas pelos métodos Doppler vascular e oscilométrico

\begin{tabular}{ccccccc}
\hline & Pressão sistólica & & & \multicolumn{3}{c}{ Pressão diastólica } \\
\cline { 1 - 2 } \cline { 5 - 6 } & PASd & PASo & & animal & PADd & PADo \\
\hline 2 & 142 & 149,6 & 1 & 106 & 89,8 \\
3 & 148 & 149,6 & 2 & 116 & 84,8 \\
4 & 141 & 123,8 & 3 & 106 & 87 \\
5 & 191 & 150,8 & & 4 & 144 & 92 \\
6 & 140 & 136,4 & 5 & 64 & 87,6 \\
7 & 177 & 160,8 & 6 & 130 & 95,4 \\
8 & 115 & 116,2 & 7 & 88 & 79,6 \\
9 & 116 & 140 & & 9 & 94 & 93,8 \\
10 & 136 & 123 & 9 & 104 & 90,4 \\
11 & 154 & 156 & 10 & 128 & 96 \\
12 & 131 & 138,8 & 11 & 108 & 91,8 \\
13 & 144 & 131,4 & 12 & 121 & 85,4 \\
14 & 134 & 124,6 & 13 & 108 & 87,2 \\
15 & 152 & 150 & 14 & 126 & 85 \\
MD & 165 & 136 & 15 & 128 & 79,2 \\
DP & 145,73 & 139,13 & MD & 111,4 & 88,33 \\
\hline
\end{tabular}

PASd: médias de pressão arterial sistólica aferidas pelo Doppler vascular; PASo: médias de pressão arterial sistólica aferidas pelo método oscilométrico; PADd: médias de pressão arterial diastólica aferidas pelo Doppler vascular; PADo: médias de pressão arterial diastólica aferidas pelo método oscilométrico; MD: média; DP: desvio-padrão. 
Tabela 3. Médias de pressão arterial sistólica e diastólica de cães de grande porte, aferidas pelos métodos Doppler vascular e oscilométrico

\begin{tabular}{cccccc}
\hline & Pressão sistólica & & & \multicolumn{3}{c}{ Pressão diastólica } \\
\cline { 1 - 3 } \cline { 5 - 6 } & PASd & PASo & & PADd & PADo \\
\hline 1 & 164 & 169,2 & 1 & 118 & 94,6 \\
3 & 160 & 143,4 & 2 & 112 & 95,2 \\
4 & 118 & 130 & 3 & 98 & 64,4 \\
5 & 108 & 181,4 & 4 & 68 & 110,6 \\
6 & 144 & 175,6 & 5 & 92 & 122,4 \\
7 & 130 & 141 & 6 & 81 & 94,4 \\
8 & 143 & 133,4 & 7 & 118 & 70 \\
9 & 108 & 135,8 & 8 & 59 & 66,6 \\
10 & 149 & 140,6 & 9 & 96 & 66 \\
11 & 138 & 133,8 & 10 & 114 & 88,6 \\
12 & 142 & 153,4 & 11 & 112 & 95,8 \\
13 & 130 & 133,4 & 12 & 106 & 70,6 \\
14 & 130 & 170,8 & 13 & 90 & 84,4 \\
15 & 140 & 146 & 14 & 108 & 109,6 \\
MD & 138 & 128,2 & 15 & 102 & 93,4 \\
DP & 136,13 & 147,73 & MD & 98,26 & 88,44 \\
\hline
\end{tabular}

PASd: médias de pressão arterial sistólica aferidas pelo Doppler vascular; PASo: médias de pressão arterial sistólica aferidas pelo método oscilométrico; PADd = médias de pressão arterial diastólica aferidas pelo Doppler vascular; PADo: médias de pressão arterial diastólica aferidas pelo método oscilométrico; MD: média; DP: desvio-padrão.

As Fig. 1 e 2 mostram os resultados obtidos, pelos dois métodos, nos três grupos de animais.

A análise estatística mostrou, por meio do teste de normalidade de Anderson-Darling, que a diferença das amostras obedecia a uma distribuição normal $(\mathrm{P}>0,05)$. Dessa forma, foi possível optar por um teste paramétrico para comparar as médias de pressão arterial sistólica aferidas pelos métodos Doppler vascular e oscilométrico, bem como as médias da pressão arterial diastólica, aferidas pelos mesmos métodos, para os três grupos. O teste escolhido para esse caso foi o t pareado, em que $\mathrm{P}>0,05$ aceita a hipótese de que ambos os métodos são equivalentes na obtenção da pressão arterial e $\mathrm{P}<0,05$ rejeita essa hipótese.

Estatisticamente, não se observou diferença entre as médias da pressão arterial sistólica aferidas pelos dois métodos, $\mathrm{P}=0,59, \mathrm{P}=0,13$ e $\mathrm{P}=0,08$, respectivamente, para os grupos I, II e III, isto é, não houve diferença entre o método Doppler vascular e o método oscilométrico na obtenção da pressão arterial sistólica. Em relação à pressão arterial diastólica, observaram-se diferenças entre as médias de pressão aferidas pelos dois métodos, $\mathrm{P}<0,02$ e $\mathrm{P}<0,01$, respectivamente, para os grupos I e II, isto é, houve importante diferença entre o método Doppler vascular e o método oscilométrico na obtenção da pressão arterial diastólica.

Estatisticamente, não se observou diferença entre as médias de pressão arterial sistólica aferidas pelos dois métodos $(p=0,128)$, ou seja, para o grupo II, não há diferença entre o método Doppler vascular e o método oscilométrico na obtenção da pressão arterial sistólica. Já em relação à pressão arterial diastólica, puderam-se observar diferenças significantes entre as médias de pressão aferidas pelos dois métodos diferentes $(\mathrm{p}=0)$, ou seja, para o grupo II, há uma importante diferença entre o método Doppler vascular e o método oscilométrico na obtenção da pressão arterial diastólica. Quanto à pressão arterial diastólica, não houve diferença entre as médias de pressão aferidas pelos dois métodos $(\mathrm{P}$ $=0,16)$, para o grupo III. 


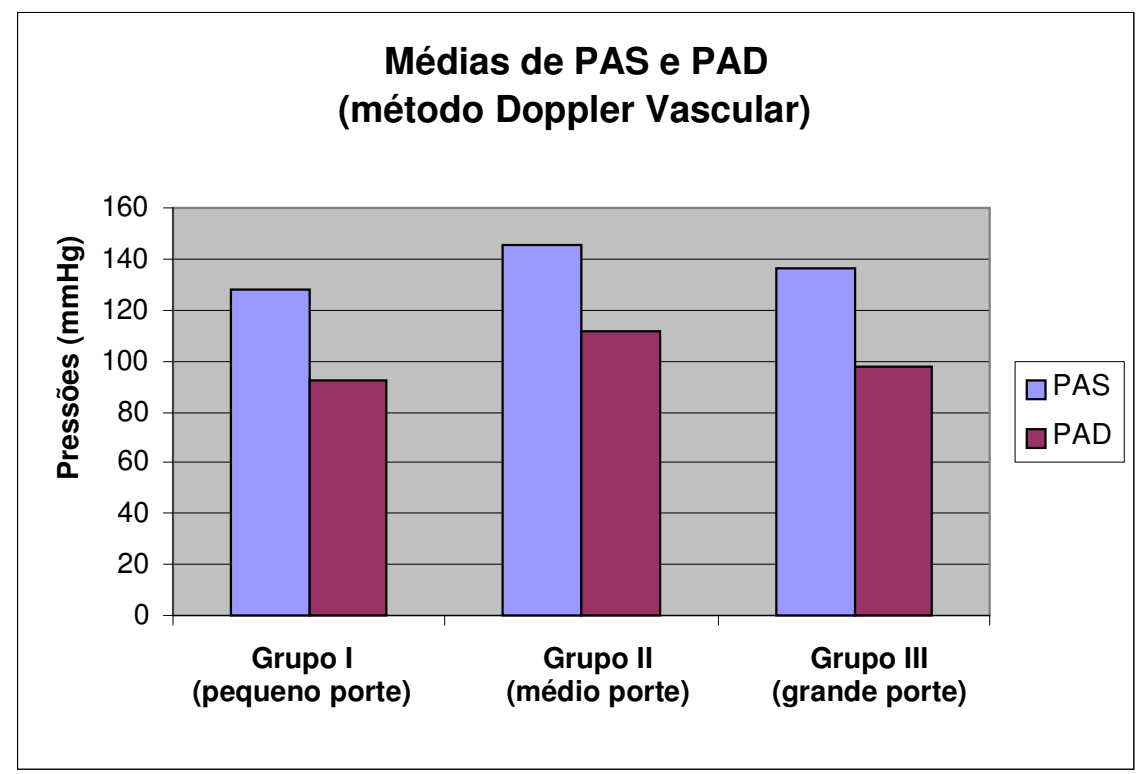

PAS: pressão arterial sistólica; PAD: pressão arterial diastólica.

Figura 1. Médias da pressão arterial sistólica e diastólica, aferidas por meio do método Doppler vascular, nos diferentes grupos.

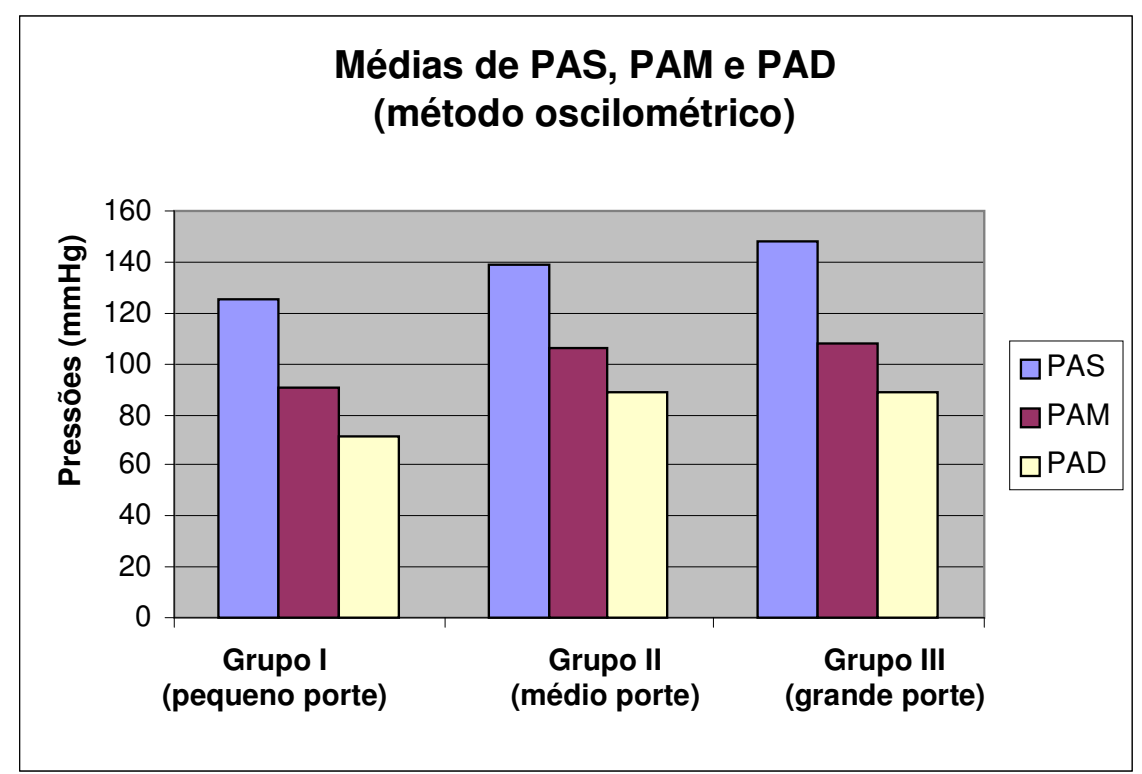

PAS: pressão arterial sistólica; PAM: pressão arterial média; PAD: pressão arterial diastólica.

Figura 2. Médias de pressão arterial sistólica, média e diastólica, aferidas por meio do método oscilométrico, nos diferentes grupos. 


\section{DISCUSSÃO}

Com base na classificação de Tilley e Goodwin (2002), observou-se que a média da pressão sanguínea sistólica e a da diastólica dos animais do grupo I, medidas pelos métodos Doppler vascular e oscilométrico, foram classificadas como discretamente elevadas, enquanto a média de pressão diastólica dos animais do grupo I, pelo método oscilométrico, foi classificada como normal.

A média de pressão sanguínea sistólica e a média da pressão diastólica dos animais do grupo II, quando medidas pelo Doppler vascular, apresentaram-se discretamente e moderadamente elevadas, respectivamente. Quando aferidas pelo método oscilométrico, apresentaram-se discretamente elevadas.

Para o grupo III, as médias de pressão sanguínea sistólica e diastólica, aferidas tanto pelo Doppler vascular quanto pelo método oscilométrico, foram classificadas como discretamente elevadas.

Ainda, de acordo com Tilley e Goodwin (2002), pode-se dizer que os animais apresentam, mais frequentemente, pressão arterial classificada como discretamente elevada, exceto no caso da pressão arterial diastólica. Além disso, os resultados sugeriram que somente não há associação entre o método Doppler vascular e o método oscilométrico para a obtenção da pressão arterial diastólica. A essas observações atribui-se o fato de que a obtenção da pressão arterial diastólica, pelo método Doppler vascular, é bastante subjetiva, uma vez que depende das impressões pessoais do examinador, ou seja, este deve estar atento às mudanças no som, quando os sinais audíveis abruptamente descem ou alteram o timbre, tornando-se abafados. Resultados semelhantes foram obtidos por Jepson et al. (2005).

Os animais de pequeno porte tendem a apresentar valores de pressão arterial sistólica, média e diastólica menores do que os animais de médio e grande porte. Larsson e D`Urso (1998) observaram resultados muito semelhantes, não somente em cães sadios, mas também em cães cardiopatas.
Ao longo da execução deste trabalho, notou-se também que o aparelho oscilométrico apresentava maior frequência de erros durante a aferição da pressão arterial em animais de pequeno porte, o que pode ter contribuído para os resultados obtidos. Observou-se que os animais do grupo I foram aqueles que apresentaram maiores níveis de estresse durante a aferição da pressão arterial (apresentavam-se muito agitados e com tremores excessivos); além disso, apenas no grupo III não se observou diferença na obtenção da pressão arterial sistólica e diastólica por ambos os métodos. Esses fatos confirmam os já observados por Podell (1992), que concluiu que técnicas indiretas para a obtenção da pressão arterial, como o método oscilométrico, são menos precisas quando a pressão sanguínea é baixa, quando há vasoconstrição ou quando ocorre movimentação excessiva do animal.

\section{CONCLUSÕES}

Conclui-se que valores confiáveis de pressão arterial sistólica podem ser obtidos tanto por meio do método Doppler vascular quanto do oscilométrico, para animais de pequeno, médio e grande porte, e que os valores de pressão arterial diastólica, obtidos pelos dois métodos, não se correlacionam devido à grande variação de seus valores, de forma especialmente marcante nos animais de pequeno e médio porte.

\section{REFERÊNCIAS BIBLIOGRÁFICAS}

BODEY, A.R.; MICHELL, A.R. Longitudinal studies of reproducibility and variability of indirect (oscillometric) blood pressure measurements in dogs: evidence for tracking. Res. Vet. Sci., v.63, p.15-21, 1997.

BODEY, A.R.; MICHELL, A.R.; BOVEEE, K.C. et al. Comparison of direct and indirect (oscillometric) measurement of arterial pressure in conscious dogs. Res. Vet. Sci., v.61, p.17-21, 1996.

BROWN, S.; FINCO, D.; NAVAR, L. et al. Impaired renal autoregulatory ability in dogs with reduced renal mass. J. Am. Soc. Nephrol., v.5, p.1768, 1995. 
BROWN, S.A.; HENIK, R.A. Diagnosis and treatment of systemic hypertension. Vet. Clin. North Am.: Small Anim. Pract., v.28, p.14811493, 1998.

CHALIFOUX, A.; DALLAIRE, A.; BLAIS, D. et al. Evaluation of the arterial blood pressure of dogs by two noninvasive methods. Can. J. Comp. Med., v.49, p.419-423, 1985.

DOUGLAS, P.S.; TALLANT, B. Hypertrophy, fibrosis and diastolic dysfunction in early canine experimental hypertension. J. Am. Coll. Cardiol., v.17, p.530, 1991.

JEPSON, R.E.; HARTLEY, V.; MENDL, M. et al. A comparison of CAT Doppler and oscillometric memoprint machines for noninvasive blood pressure measurement in conscious cats. J. Feline Med. Surg., v.7, p.147$152,2005$.

KITTLESON, M.D.; OLIVER, N.B. Measurement of systemic arterial blood pressure. Vet. Clin. North Am.: Small Anim. Pract., v.13, p.321-336, 1983.

LARSSON, M.H.M.A.; D'URSO, F. Determination of arterial pressure by indirect oscillometric method in healthy, cardiac and nephropathic dogs. In: WORLD CONGRESS SMALL ANIMAL VETERINARY ASSOCIATION, 23., 1998, Buenos Aires. Anais..., Buenos Aires, 1998. v.2, p.766.
MARCO, V.D.; LARSSON, C.E. Hipertensão secundária ao hiperadrenocorticismo hipofisário canino. Rev. Bras. Cienc. Vet., v.7, supl., p.80, 2000.

PODELL, M. Use of blood pressure monitors. In: BONAGUARA, J. D. Kirk's current veterinary therapy XI: small animal practice. Philadelphia: W.B. Saunders. p.834-837, 1992.

PORCIELLO, F.; BIRETTONI, F.; CONTI, M.B. et al. Blood pressure measurements in dogs and horses using the oscillometric technique: personal observations. Vet. Res. Comm., v.28, p.367-369, 2004.

STEPIEN, R.L.; RAPOPORT, G.S. Clinical comparison of three methods to measure blood pressure in nonsedated dogs. J. Am. Vet. Med. Assoc., v.215, p.1623-1628, 1999.

TILLEY, L.P.; GOODWIN, J.K. Manual of canine and feline cardiology. 3.ed. Philadelphia: W.B. Saunders, 2002. p.337-344. 